Vaidyanathan, K., Vasipalli, P., and Singh, S.C. (2018). "Building a Lean Culture into an Organization." In: Proc. $26^{\text {th }}$ Annual Conference of the International. Group for Lean Construction (IGLC), González, V.A. (ed.), Chennai, India, pp. 1101-1110. DOI: doi.org/10.24928/2018/0439. Available at: www.iglc.net

\title{
BUILDING A LEAN CULTURE INTO AN ORGANIZATION
}

\author{
Vaidyanathan Kalyan ${ }^{1}$, VasipalliPratap ${ }^{2}$, and Singh, Srikanth Chouhan ${ }^{3}$
}

\begin{abstract}
The Indian construction industry is in need for a change; a change that can help its people and organizations discard the conventional project management approach of 'Command and control'; a change that can create better collaboration between project teams and reduce blame games. Lean construction and the Last Planner System ${ }^{\mathrm{TM}}$ is a potent and proven solution that offers the change.

This paper suggests/proposes an approach for an organizational lean transformation. The approach is based on real life experience of the authors working with both Owner and Contractor organizations. The approach is based on building experience in lean tools at the site level that can be scaled across projects as a bottoms-up model. This is complemented with a top down approach that builds awareness, empathy, and knowledge at the leadership level. Together, the goal is to build capability and confidence in the organization to adopt and adapt lean into the organization. This paper explains in detail about the program and evolution of it from Indian experience.

The authors will share their experience and their learning making the change in organizations. They will share the successes and the limitations of the approach.
\end{abstract}

\section{KEYWORDS}

Lean construction, Indian Construction, Lean Implementation, Last Planner System, Organisational Culture.

\section{INTRODUCTION}

India is in the midst of immense and rapid infrastructure growth (Raghavan, Satyanarayana., 2014) and the construction industry has yet to fully realize the impact of this. While business volumes will grow, other dynamics will enter like increased competition, reduced margins, shortage of labor etc. The large and medium size stakeholders (Clients, PMC's, Contractors) in the Indian construction industry are fast

1 CEO, Nadhi Information Technologies Pvt. Ltd., 22 Venkatraman Street, T. Nagar, Chennai 600017. India, +9197910 41483, kalyanv@ nadhi.in

2 Consultant, Nadhi Information Technologies Pvt. Ltd., 22 Venkatraman Street, T. Nagar, Chennai 600017. India, +9198841 12759, pratapv@ nadhi.in

3 Senior Consultant, Nadhi Information Technologies Pvt. Ltd., 22 Venkatraman Street, T. Nagar, Chennai 600017. India, +91 86527 00139, srikanths@ nadhi.in 
realizing that the current project management practices are inadequate to contend with these shifting market dynamics, the rapid pace of works, and still operate profitably. As a result, everybody is on the look-out for systems that can give better control of the projects, improve operational efficiency and profitability so as to compete with (other key) global stakeholders. This paper proposes an approach that an organization aspiring for such change can adapt and adopt for organizational lean transformation for improved operational efficiency.

From the authors' real-life experiences, it was observed that, more than a few organizations have identified lean as the missing ally to operate at better efficiency and profitability. But, given the rapidity and need for speed of change, organizations are trying to do independent isolated pockets of experimentation on lean. For instance, there are more than a few instances of project/site based lean engagements to bring Last Planner System, Kaizen and Value Stream Mapping et.al. There are other instances where the organizations have come-up with HR Learning \& Development initiatives for training the top management on Lean and to create awareness amongst the senior personnel. More recently, there have been rapid advancements in construction technology and there are instances where technology also being tried in addition to process changes to bring in better operation efficiency. IT departments are bringing in mobility, 3D parametric modelling solutions, and digitization initiatives under the BIM (building information modelling) initiatives to improve business processes and project operations. Although these isolated attempts on embracing lean into their organizations have yielded some positive impact, we hypothesize that, they are unable to see the transformative impact that they are expecting. This is because, we postulate that they could not formulate a corporate strategy or a holistic approach for organizational lean transformation.

The remainder of the paper is structured as follows. The next section will explain the formulation and basis of the hypothesis. Then we describe two case studies based on experience of working with a couple of Indian companies and use it as the basis to explain the methodology for the proposed approach. In the last section, we will have some discussion describe a possible corporate strategy for the transformation and then conclude with scope for future work.

\section{HYPOTHESIS}

Decision makers in the Construction Industry in India are now exposed to lean construction for the past few years. They understand and agree that lean construction would be a vital strategy to improve the performance of construction and create substantial improvements for firms that are adopting it (Ballard and Howell 2003).

The authors' hypothesis is that, isolated attempts can lead to sub-optimal results and probably question the efficacy or applicability of lean. What is needed is, a somewhat of holistic approach/strategy that is long term and comprehensive methodology for incorporating/inculcating lean tools, processes, and complementing technologies along with the culture into the organization; to make it sustainable and yield substantial benefits. There are references from literature that support this hypothesis. For instance, (Leonova, 
Ballard and Gehbauer, 2017) mentioned that if the success of lean adoption is suboptimal, then we need a discussion about improved strategies.

\section{METHODOLOGY}

The authors have been engaged on lean consulting engagements for projects in India for nearly a decade now. They have experience working across infrastructure (Vaidyanathan et al 2015), residential real estate (Vaidyanathan, Pratap 2015), and commercial real estate projects (Vaidyanathan et al 2016) both with Owners and Contractors. A typical engagement would last atleast a year with the authors acting as lean coaches bringing about the adoption of the Last Planner System, Value Stream Mapping and other such lean tools to transform the culture of managing the projects to be more transparent, collaborative and problem solving based from the conventional project monitoring based on cost-based targets leading to blame game when targets are missed. Metrics and output measured on a couple of the engagements are documented in previously published case studies listed above. Based on their experience and understanding on working on nearly twenty such engagements, the authors are proposing a two-pronged approach to adopting lean at an organization level with complementary process and technology strategy. A Bottom-Up approach of implementing lean tools at the site to build capacity and collaboration between site teams that is complemented by a Top-Down approach that builds awareness to management drive continuous improvement initiatives. The approach is explained with two independent case studies that are elaborated below. The first case study is of an EPC contractor amongst the top ten contractors in India with operations pan India. The second case study is of a real estate developer working on large residential projects in Mumbai region, India.

\section{BOTTOM-UP APPROACH: CAPACITY BUILDING}

The intention of the program is to teach and inculcate the basics of lean and lean tools. Tools such as the Last Planner System ${ }^{\mathrm{TM}}$ (LPS), Value Stream Mapping (VSM) and work sampling to the site personnel at various sites in a scalable and repeatable manner. Besides teaching the team on lean, the program is aimed at a cultural change amongst the site team. The following is a case study of implementing this for an organization.

The organization, in question, is one of the top ten EPC contractors in India. It has several regional offices spread across India. It has a conventional project monitoring and control set up, driven in theory by the PDCA logic. The planning system is focussed more on the master schedule, target completion schedule and the monthly plan. The execution team is expected to deliver on the monthly plan. The plans are typically difficult to achieve and not owned by the last planners and hence the plans tend to fail. Filling this ground level void of making the plans work in real time is what LPS is meant to achieve.

The organization, drawing from its past experience with implementing lean and LPS (Vaidyanathan, Pratap 2015), decided to implement lean construction principles across a region on a pilot basis and then depending upon the degree of success, scale it to the whole organization in the long term. They hired a lean coach that they had the past experience with to evolve a strategy to achieve the goal. The initial plan was to employ the lean coach in each site for a three-month duration. The objective of the coach was to 
get the site team to learn and adopt the lean process, specifically LPS for collaborative planning and VSM for productivity enhancement or wastage reduction and to tailor the lean processes and systems to the specific project requirement (if any) for planning and controlling. In short, the purpose was to mentor the team, institutionalize the process and repeat the process. The program was implemented for about five projects over a period of fifteen months with each Project having different deliverables with different set of challenges. The program was rolled-out to three residential township projects, a commercial facility, and to an industrial project.

LPS was designed to improve workflow reliability by continuously aligning what will be done on projects with what should be done through collaborative planning (Ballard and Howell, 1997). From the phase schedule, the site team was to identify the fifth week's (rolling Look ahead Plan) major activities. Then they were to identify the constraints or make ready processes for each of the activities. With that a weekly work plan is created which a list of commitments (WILL do) from the last planners. Daily monitoring and controlling is done on the commitments. Adherence or misses to commitments are measured to calculate percent plan complete (PPC) and failures lead to learnings and taking corrective actions using VSM or other intervention techniques. The achieved production during the week (successes) is tied back to the phase schedule and master schedule to ensure that contractual milestones are not being missed. In this sense, an alignment between planning and execution from top to bottom and bottom to top was created. Production was emphasized over productivity and cycle time was measured as the KPI rather than revenue (or cost) for the organization.

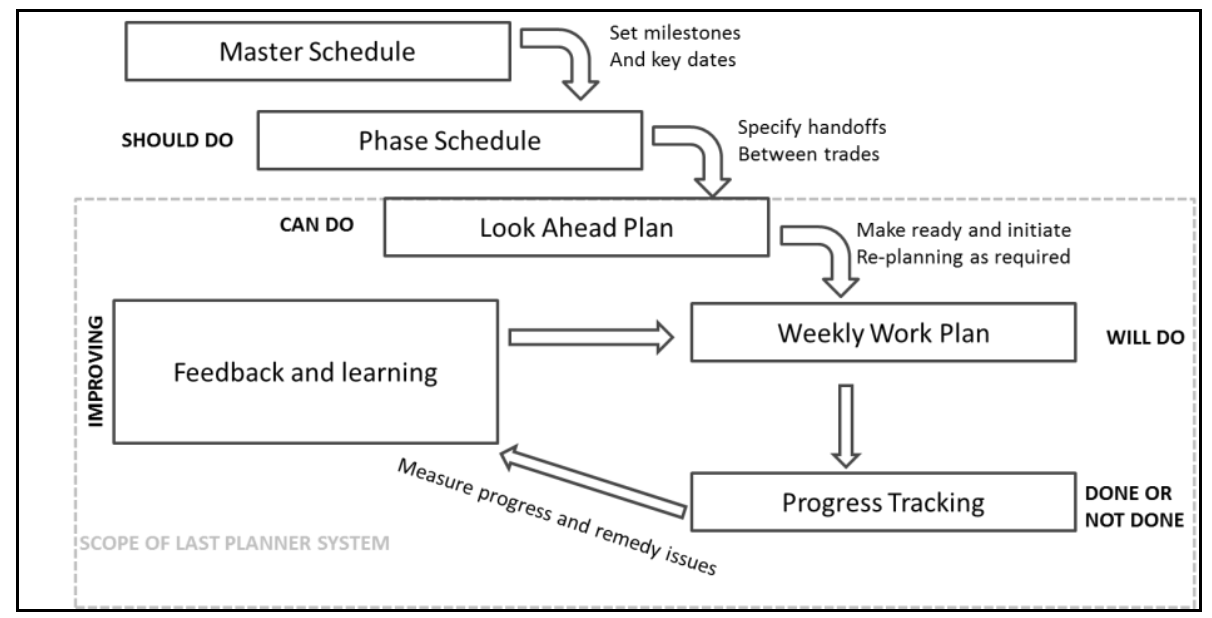

Figure 1: Last Planner System

The Lean training program at each site would start with a lean and LPS awareness workshop for the site team with top management in attendance. Over the next week or two, the lean consultant had to observe the AS-IS planning process and the site conditions, so as to come up with a roadmap for rolling out the project specific LPS. Thereafter, a LPS introduction workshop with simulation games and a working planning session would be conducted by the lean consultants to help the last planners and the whole site team to understand the LPS methodology. 
The program got much needed impetus from the second site where, when the site team was able to bring the slab cycle down from 12/13 days to a consistent 8 days' time as shown in the figure 2 . And after the third site, the three-month implementation plan was standardized and became a standard operating procedure. The idea was to mainly teach the LPS and VSM techniques to the sites, but in the process also create excitement within the teams by drawing on their problem-solving abilities and creating commitment and ownership within the teams to improve productivity and throughput. This also had the desired effect of helping sites and site planning and management take better ownership and adopt the lean processes and to learn and control the project using LPS.

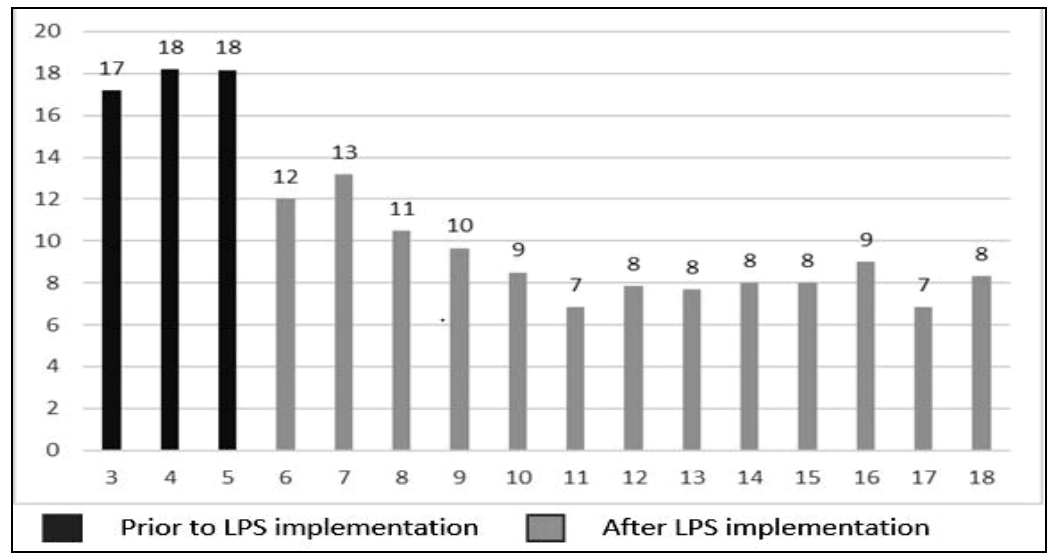

Figure 2: Pour Cycle Trend at Second Site

Project reviews by management was also changed to encourage adoption of lean principles. The monthly project reviews at the head office started to involve discussion on performance indices based on lean parameters. These included:

- Production metrics rather than productivity metrics

- Discussion around constraint analysis and removal rather than delay justification

- PPC and interventions done to avoid repeat of the top 3 delay reasons

At the end of the program, the methodology of implementing Last Planner System and conducting meetings were all standardized. The adoption of lean at the sites improved and it had the cascading effect of other projects and other sites asking to adopt similar processes and techniques.

\section{TOP-DOWN APPROACH: DRIVING INITIATIVES}

It is a known fact and people accept that adoption of lean requires changes in attitudes, thinking and behavior (Leonova, Ballard and Gehbauer, 2017). And, there is a need for creating awareness that can drive the change and develop the drivers. Lean construction must be accompanied by a conscious and consistent strategy and championed by senior level managers (Mossman 2009). The organization in question, is a residential and commercial real estate developer with projects primarily being done in Mumbai, India. They are trying to scale the organization for the next level growth. As a HR initiative for 
their next generation leaders to champion the growth using lean, the organization partnered with a lean consulting firm and a lean leadership training program was designed. The program's goal was to create lean awareness and impart basic knowledge of lean tools amongst the senior managers and the top management. The hypothesis was that rather than simply giving them theoretical knowledge on lean and various lean tools, they will get to appreciate it better and realize its potential if they get to apply them themselves to processes in the business or to their job routines. And once they realized the potential of lean (and the specific tools), they will be more committed change drivers that will drive change within the organization for the long term. The following is a case study of implementing this for an organization.

The organization is a forward-thinking entity that always has an eye towards leadership training programs for management personnel to keep them motivated. The organization partnered for one such training program with the consulting firm. The program was a HR initiative as part of their learning and development program that the participants had to undergo. In this case, the participants happened to be typically heads of various departments from sales, marketing, customer service, and project operations like contracts, procurement, field execution, planning, information technology etc. The goal was for each of them to learn about the various functions in the company and how the various functions are inter-connected and operates in a cross-functional learning environment using lean as the underlying theme. The program was designed as a oneyear continuous learning and application program. Each quarter of the year, one or two topics of lean was introduced to the teams in a learning session. During the quarter, the participants were to apply the learning in a practical work scenario as part of application (and development of the individual/team/organization). End of the quarter, the participants had to present their experience between themselves and to the management. While team had the flexibility to apply their learnings on any of the organization's process or functions, the participants were grouped into four teams and each team was given a broad theme and encouraged to pick application of lean techniques on the theme assigned to them. Following are the teams and their initiatives:

- Quality team - First time right

- Cost optimization team

- Customer experience enhancement team

- New Product development team

Each team had an average of about seven members. And each team was consciously heterogenous with participants from various business functions and departments described above. A kick-off session was conducted where the whole of the teams was inducted on lean. The curriculum for the year was as follows:

- Learning to see - Understanding waste and value stream mapping

- Collaborative planning - Understanding teamwork and Last planner system

- Continuous improvement - Kanban, 5S, poka yoke

- Technology enablers - BIM, project controls 
The structure of the course during the quarter followed the following structure. Each quarter, a one or two-day workshop was conducted to introduce the next lean topic as outlined above. Each team had to then come up with a proposal of the application of the lean topic within their daily routine based on the theme assigned to them. During the quarter, the lean consultant would work with individual teams on a weekly basis to ensure that each team was making progress on their proposal. Monthly reviews were done internally within the teams to encourage each other as needed. At the end of the quarter, each team had to present the results of their proposal to top management who acted as mentors. Successful proposals for improvement that were made to management was implemented in a pilot mode and if found successful, was operationalized into the business. This followed introduction of the new lean tool for the next quarter and a repeat of the process ensued.

For instance, the Quality team could choose to see how to ensure tile installation is done right without re-work as part of their first quarter experiment. But this team had members from execution and also from procurement, billing, sales etc. The nonexecution team would bring out-of-the-box ideas on how to improve an execution function. In the process, the expectation was that, the team members would get practical experience on adapting and adopting the lean tools while also learning the functions of other departments at a working level of detail that they otherwise would not have an opportunity to know about in the "busyness" of their daily routine.

The cross-functional teams had the desired benefit of each department head learning to empathize with the functions of their peers, heads of departments of other functions in a "fun filled", "classroom learning environment". To finish up the earlier example, the team studied the tiling process from design to execution and came up with standards for design, procurement, and execution to ensure that quality of execution was at its highest. To take another example, execution personnel should know how and why bill processing takes "time" in the Billing \& Accounts department. At the same time, billing personnel get to appreciate the difficulties of measuring productivity and work output at the sites under harsh site conditions and with sub-contractors of varying education and awareness levels and give information in exactly the formats desired by the information technology systems designed by the accounts department. Each learnt to give and take a little to collectively ease the pain and friction in bill processing. It should be noted that these heads of departments are expected to take the organization to the next level and this classroom environment gave them a safe space to become friends and teammates. The hope is that once both get back to their daily routines, they will be able to call on their friendship at difficult times. The team came up with innovation campaign and got participation from the entire company to contribute ideas for innovation.

With each passing quarter, it was evident that the teams showed improvement in leadership skills, coordination skills, analytical skills, proposal writing skills, thought process, implementation planning, etc. to name a few. Most importantly the teams developed an eye for identifying waste, appreciated the culture of collaboration and teamwork, and created an environment for mutual trust and respect and developed empathy for each other's work and process. 
At the end of one year, several improvement initiatives were piloted and successful ones were operationalized. The top management of the organization was very pleased with the learning environment that was created in the organization. In fact, the organization went on to win several awards for this initiative under the learning \& development category of HR awards. It has been a couple of years since the program has been completed, but till date the improvement initiatives are created and driven periodically by these next generation leaders.

\section{THE PROPOSED APPROACH}

The discussion on each of the case studies show that individually, both had the desired effect of bringing change in the organization. In the first case, the site teams were "owning" the execution and driving improvement. But for it to become a "habit", what the authors had to do was make management change the review mechanism. If that were not done, and if management metrics continued to be the old metrics (productivity, cost, revenue, profitability etc.), the risk of the site teams falling back into old habits existed. In that sense, top management and site teams had to be aligned. But if the top management was not convinced of the potential of the lean techniques, their willingness to change is not very forthcoming.

This is where the second approach works. Here, the top management is given time and space to learn and appreciate the potential of individual lean tools and the lean philosophy and culture in general. That gives them confidence to drive change in the sites in a continuous improvement manner. In this case, if the site teams did not have the capacity to adapt themselves to the new tools and metrics (production, constraint analysis, intervention and problem-solving attitude etc.), then the top management is unable to drive the change initiative. Here the bottom up capacity building is required to get the site teams to organically adopt the lean process, plan using LPS and problem solve using VSM.

In short, each of the approaches was sort of incomplete without the other although independently, they were valuable. That leads to our proposed two-pronged approach. The approach is for both of the above to be done in tandem - have site teams learn the lean tools, LPS, VSM and build capability and capacity from the bottom up and in parallel, have top management drive initiatives for change and improvement after having realized the potential of lean and lean tools. This combined strategy, as shown in Figure 3, will have the desired effect of changing the way projects are being managed from the conventional approach to a more lean enabled production based approach. Now, the people at all levels would able to appreciate and talk in the same language and terminology of lean.

The project review mechanism/conversations would then change and metrics measured would not be conventional metrics of cost, but also lean metrics like production. The monthly or even the weekly review meetings should involve discussions on look ahead constraint analysis that is more a forward-looking problem (and delay) avoidance approach rather than monitoring delays (and pegging delays to external and internal stakeholders). Healthier discussions are possible around intervention techniques based on 
PPC metrics. In a sense, the whole discussion has shifted from a negative spiral of delay analysis based on lag indicators to problem avoidance based on lead indicators.

That leaves the information technology solutions used to manage the project information. In this new environment, technology should be the enabler for the transformation of an organization into a lean organization. It has to work in this new environment of proactive information exchange, open collaboration across business functions, and measure KPIs and metrics driven by lean metrics. In short, it has to complement the lean process. This would justify the adoption of tools including 3D modelling, integration of disparate information sources like finance and auditing (ERP) systems, scheduling systems, document management systems, and building information modelling (BIM) authoring tools. The technology and process together will complement to make an approach for integrated project controls driven with on a base built on lean culture making the whole organizational strategy a holistic one.

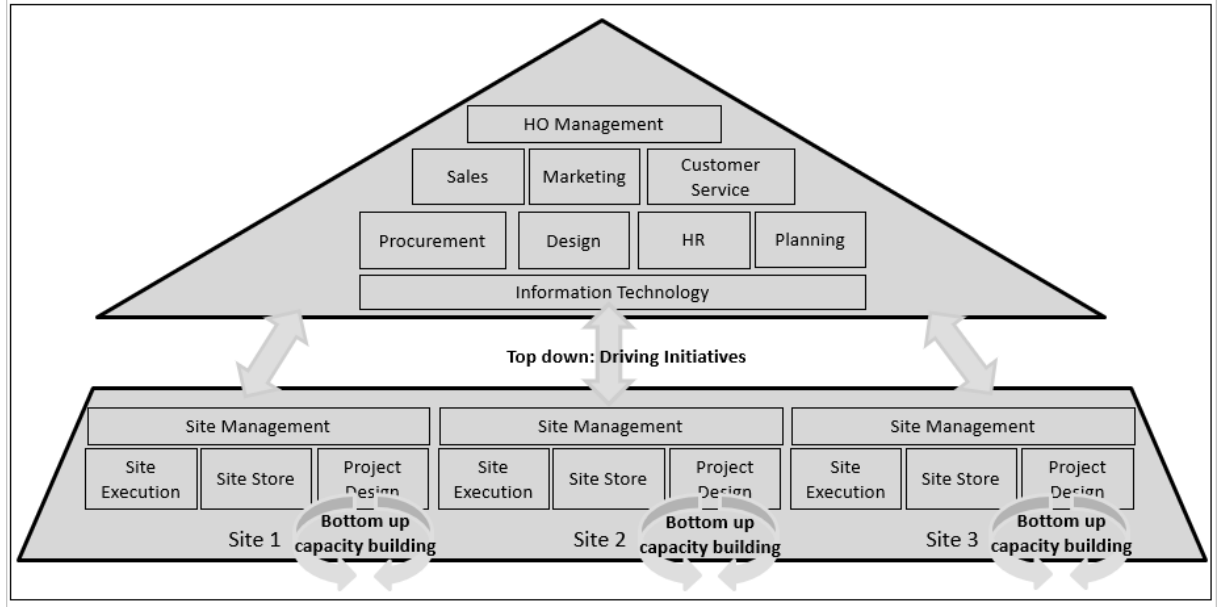

Figure 3: Lean Transformation Strategy

\section{DISCUSSION}

The authors feel that further work is required to measure the effectiveness of the transformation through organization qualitative and quantitative KPIs. Indicative ideas are metrics like employee satisfaction, business profitability, resilience to changing market dynamics etc. And a lean transformation maturity model that can be measurable should be arrived at.

There have been references and discussions about the requirement of a framework for an organizational lean transformation in literature. For instance, (Leonova, Ballard and Gehbauer, 2017) has discussed about industry transformation and has outlined strategies and an approach to lean transformation of the construction industry. (Nesensohn 2017), proposed a Lean Construction Maturity Model to benchmark the lean maturity of any organization. But they do not provide a practical approach on how to achieve the transformation. The authors here, based on their experience and benefits that customers have realized, propose a simple and practical approach for an organization to adopt in its journey to lean transformation. The approach needs validation and further development 
and refinement, something that the authors hope to engage in with their next assignments and report on in the future.

\section{CONCLUSION}

An approach has been shown that organizations can adopt as a strategy to bring lean transformation to their organizations. The approach consists of building capability in the teams from the bottoms up that is complemented by having management drive continuous improvements from the top down. Each approach complements the other and together they help migrate the organization from its current state to a leaner organization that drives continuous improvement. Over time, this would build a culture of lean in the organization. The approach is practical and simple. It is prescriptive, yet adaptive to various organizations. The approach is generic enough for adoption both with Owners and Contractors. The authors are planning to apply the framework in future client engagements in the near future and will report on their learnings and findings in the near future.

\section{REFERENCES}

Ballard, Glenn (2000). "The Last Planner", Lean Construction Institute White Papers.

Ballard, G. and Howell G.A. (2003). "Lean project management." Building Research \& Information, 31 (2) 119-133.

Howell, A. Gregory, (1999). "What is Lean Construction - 1999" Proceedings of IGLC-7, University of Berkeley, CA

Leonova, M., Ballard, G. and Gehbauer, F. (2017). "Strategies that can help Transform Construction Industry" Proceedings of IGLC-25, 2017, Heraklion, Greece.

Mossman, A. (2009). "Why Isn't the UK Construction Industry Going Lean With Gusto?" Lean Construction Journal, 24-36.

Nesensohn, Claus. (2017). "A Lean Construction Maturity Model for Organization" Proceedings of IGLC-25, 2017, Heraklion, Greece, pp. 357-363.

Raghavan, N., and Satyanarayana, K. N., 2014. "Implementing Lean Concepts on Indian Construction Sites - Organisational Aspects and Lessons Learned" Proceedings of IGLC22, June 2014, Oslo, Norway

Rother, M. and Shook, J. (2004). "Learning to See - Value-Stream Mapping to Create Value and Eliminate Muda". Lean Enterprise Institute.

Vaidyanathan, Kalyan, Nandakumar, M., Rudra, Avik, Raman, S. and Mundoli, Ravi S. (2015). "Learnings from the Application of Lean Principles for 4X300 MW Thermal Power Plant in India", First Annual Conference of Indian Lean Construction Conference, February 5-7, 2015, Mumbai India, pp 316 - 327.

Vaidyanathan, Kalyan., Reddy, Pratap., Yamgar, Smita., and Dhekale, Rajendra (2015). "Learnings from Application of Last Planner in a Residential Project" First Annual Conference of Indian Lean Construction Conference, February 5-7, 2015, Mumbai India, pp 316 - 327.

Vaidyanathan, Kalyan., Mohanbabu, S., Sriram, P., Rahman, Safiyur., and Arunkumar, S., (2016). "Application of Lean Principles to Managing Construction of an IT Commercial Facility - An Indian Experience" Proceedings of IGLC-24, 2016, Boston, USA 